\title{
Tomographic measurement of buried interface roughness
}

\author{
Misa Hayashida ${ }^{1}$, Shinichi Ogawa ${ }^{2}$, Marek Malac ${ }^{1,3}$ \\ ${ }^{1}$ National Institute for Nanotechnology (NINT), Edmonton, Alberta, Canada. \\ ${ }^{2}$ National Institute of Advanced Industrial Science and Technology (AIST), Tsukuba, Ibaraki, Japan. \\ ${ }^{3}$ Department of Physics, University of Alberta, Edmonton, Alberta, Canada.
}

Interface roughness of buried layers in nano-meter size devices often degrades performance of the devices because the roughness of the interfaces might affect expected growth of consequent layers during the device fabrication. To characterize the roughness of an exposed surface, atomic force microscope (AFM) has been widely used. On the other hand, when the interfaces are buried by additional device layers, it is impossible to measure the interface roughness by AFM. In this study, we present quantitative measurement of buried interface roughness by electron tomography using a STEM. The presented method allows to characterize interface roughness of the devices with a resolution of 1 nm.

Figure 1 shows a multilayer rod-shaped sample of a stacked $\mathrm{SiO}_{2}-\mathrm{W}-\mathrm{SiO}_{2}$ on $\mathrm{Si}$ substrate structure used to characterize the roughness. An upper $\mathrm{SiO}_{2}-\mathrm{W}$ interface exhibits larger roughness than a lower $\mathrm{W}-\mathrm{SiO}_{2}$ interface. A cross sectional rod-shaped sample was fabricated by an FIB method. Nano-dot fiducial markers for accurate alignment of the tilt series were fabricated by SEM with a gas injection system [1]. The tilt series was acquired using annular dark field STEM mode of a Hitachi HF-3300 TEM / STEM with cold field emission source and MAESTRO computer control system [2]. The tilt step was $3^{\circ}$ over the entire $\pm 90^{\circ}$ tilt range eliminating the missing wedge problem. Ten images were collected at every tilt with 5 us dwell time to decrease effect of sample drift. All the images were summed after compensating each image for drift using standard cross correlation alignment. To evaluate effect of shot noise, 3, 5, 7 and 10 images were summed at each tilt resulting in four tilt series with different electron dose. Images were 512 X 1024 pixels with $0.47 \mathrm{~nm}$ pixel size. All four tilt series were reconstructed using Filtered Back Projection (FBP).

To determine positions of the interfaces, median filter, low pass filter, Sobel filter and binary filter were applied to the slice images as shown in Figure 2. Then, the positions of interfaces between black (digital 0 ) and white (digital 1) were detected from the each $\mathrm{Z}$ slice. Approximately $7 \times 10^{4}$ points were detected in every tilt series. A plane was determined as it minimized the RMS distance from the interface for all points. Standard deviation of distances from this plane was taken as "interface roughness". The same processing was applied to all four tilt series.

Figure 3 shows measured roughness decreases with increasing electron dose because the signal to noise ratio is increasing with electron dose and the image processing works more accurately. The measured roughness tends to an asymptotic value with increasing electron dose. The four series allow us to extrapolate the measured data to the asymptotic value, which in this case, is $\sigma \sim 1.05 \mathrm{~nm}$. To verify the performance of the data processing step, we also characterized roughness of computer generated test data and it showed a good agreement between the known input roughness and the measured roughness.

[1] M. Hayashida, et. al., Ultramicroscopy, Vol 144, (2014), 50-57 
[2] M. Bergen et. al. Microscopy and Microanalysis 19 (S2) (2013), 1394-1395.

Financial support of Visiting Researcher grant from Alberta Innovates Technology Futures is gratefully acknowledged. This work was done when the first author worked at her earlier affiliation, AIST.

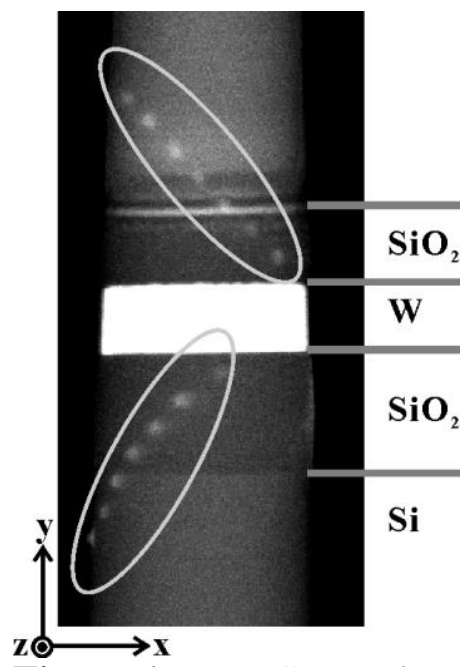

Figure 1. ADF STEM image of test sample for evaluation of interface roughness. Nano-dot fiducial markers are marked by two ellipses. (a)

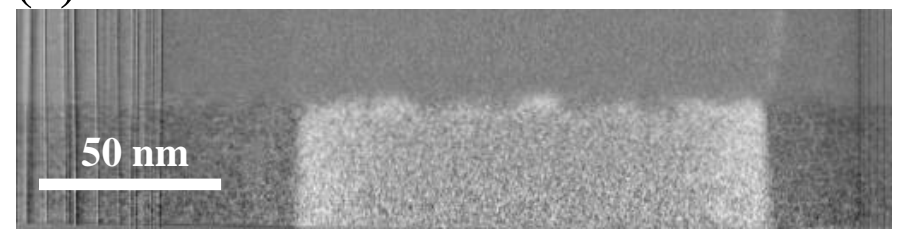

(b)

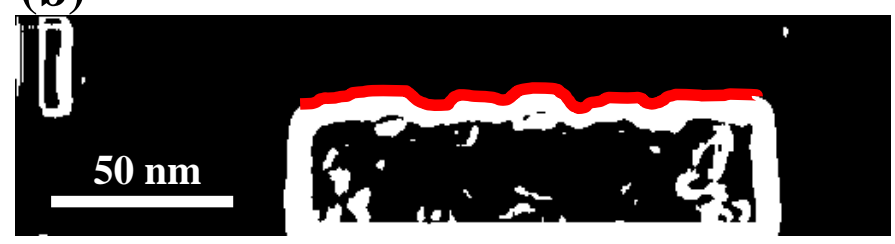

Figure 2.

(a) Z slice image of reconstructed volume

(b) Filtered image of (a). The red line is the detected interface between $\mathrm{SiO}_{2}$ and $\mathrm{W}$ layer.

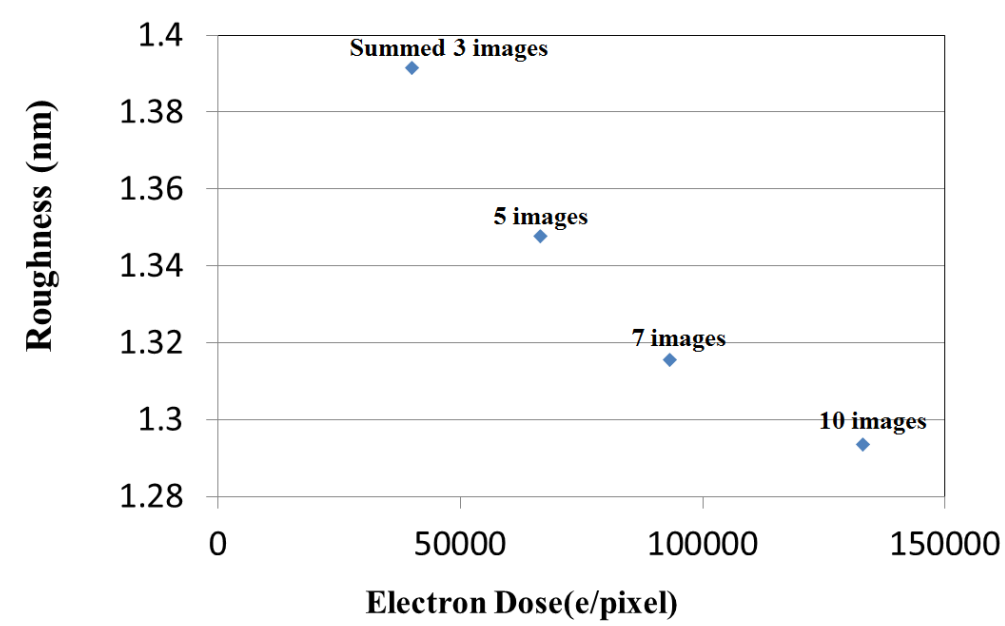

Figure 3. The measured roughness depends on signal to noise ratio (irradiation dose) and can be extrapolated to an asymptotic value of $\sigma \sim 1.05 \mathrm{~nm}$ at a very high dose. The horizontal axis is the incident number electrons per pixel for the entire $\pm 90^{\circ}$ series. 